\title{
SURVIVING VICE-PRESIDENTS
}
G. D. BIRKHOFF,
G. A. MILLER,
H. F. BLICHFELDT,
E. H. MOORE,
G. A. BLIss,
Frank MORLEX,
Oscar Bolza,
F. R. MoULton,
E. W. Brown,
W. F. OsGood,
Florian Cajori,
A. B. CoBLe,
J. L. COOLIDGE,
JAMES PIERPONT,
Charlotte A. Scott,
D. R. CuRTiss,
P. F. SMITH,
VIRGIL SNYDER,
L. E. Dickson,
W. E. STORY,
L. P. Eisenhart,
HENRY TABER,
H. B. Fine,
E. B. VAN VleECK,
T. S. FISKE,
Oswald Veblen,
JAMES HARKNESS,
H. S. White,
M. W. HASKELL,
E. J. WILCZYNSKI,
E. R. HEDRICK,
E. B. WILson,
J. I. Hutchinson,
R. S. WOODWARD,
EdWARD KaSNer,
Alexander Ziwet. 